\title{
Neurogenic tumour of the orbit in a patient with idiopathic chronic inflammatory demyelinating polyneuropathy
}

\author{
Bik Ling Man, Yat Pang Fu
}

Department of Medicine and Geriatrics, Tuen Mun Hospital, Hong Kong, Hong Kong

\section{Correspondence to} Dr Bik Ling Man, beli_man@yahoo.com

Accepted 10 August 2014
CrossMark

To cite: Man BL, Fu YP. BMJ Case Rep Published online: [please include Day Month Year] doi:10.1136/ bcr-2014-205349

\section{DESCRIPTION}

A 49-year-old man had progressive blurring of vision in the left eye for 1 year. He was seen by ophthalmologist and MRI orbit showed left intraconal orbital lesion extending around the opening of superior orbital fissure and optic canal which is suspicious of neurogenic tumour (figure $1 \mathrm{~A}-\mathrm{C}$ ). $\mathrm{He}$ was scheduled for a follow-up MRI scan before operation. He was then admitted for numbness and weakness of limbs for a few months. Physical examination showed visual acuity was finger counting at 1 feet on his left eye and left relative afferent pupil defect. There was wasting of muscles of his hands and legs, the upper limb power was Medical Research Council (MRC) grading 5-/5 and the lower limb power was MRC grading 5-/5 proximally and $3 / 5$ distally. There was reduced temperature and pain sensation in his feet up to the knee level and the jerks were hyporeflexic. Cerebrospinal fluid examinations showed white cell count $<1 / \mathrm{mm}^{3} . \quad(<5)$, protein $1.01 \mathrm{~g} / \mathrm{L} \quad(0.15-0.4)$, glucose $4 \mathrm{mmol} / \mathrm{L}$ (2.2-3.9). A nerve conduction study showed prolonged distal latencies and reduction in conduction velocities of the median, ulnar, tibial and peroneal nerves. The amplitude of the compound motor action potential (CMAP) was reduced in the right tibial, left median and both peroneal nerves. There were possible conduction blocks, defined by significant reduction in the CMAP area according to the American Association of Neuromuscular \& Electrodiagnostic Medicine criteria, ${ }^{1}$ in the right median nerve below the elbow, both ulnar nerves below the elbow and left tibial nerve below the popliteal fossa. The sensory nerve action potentials were not recordable (table 1). MRI of the cervical spine and plexus showed a diffuse thickening of the nerve roots (figure 2A) and bilateral brachial plexuses (figure 2B). Sural nerve biopsy revealed myelin sheath damage affecting both the large and small fibres. There was inflammatory infiltration of the perivascular connective tissue of the perineurium. Extensive workup for secondary causes of chronic inflammatory demyelinating polyneuropathy (CIDP) including blood glucose, autoimmune markers, immunoglobulin pattern, serum protein electrophoresis and paraneoplastic panels was unremarkable. He was diagnosed with idiopathic CIDP and was given high-dose oral prednisolone $(1 \mathrm{mg} /$ $\mathrm{kg} /$ day). His limb power improved with treatment.

Common causes of neurogenic orbital tumours include pilocytic astrocytoma, meningioma, schwannoma and neurofibroma. ${ }^{2}$ Association of CIDP with neurogenic tumour of the orbit has not been previously reported. As these two conditions are two different entities, the association is most likely incidental.

\section{Learning points}

Common causes of neurogenic orbital tumours include pilocytic astrocytoma, meningioma, schwannoma and neurofibroma.

- Association of chronic inflammatory demyelinating polyneuropathy with neurogenic tumour of the orbit has not been previously reported.

- As these two conditions are two different entities, the association is most likely incidental.
A

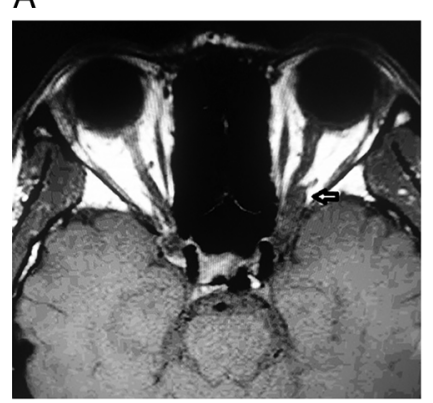

B

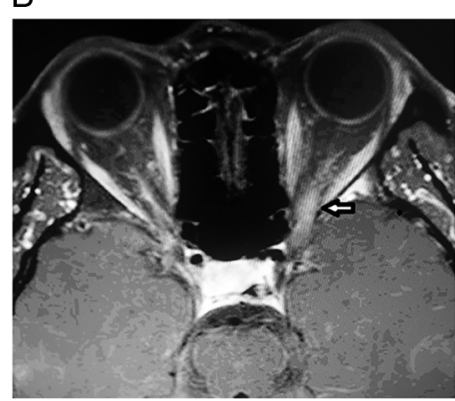

C

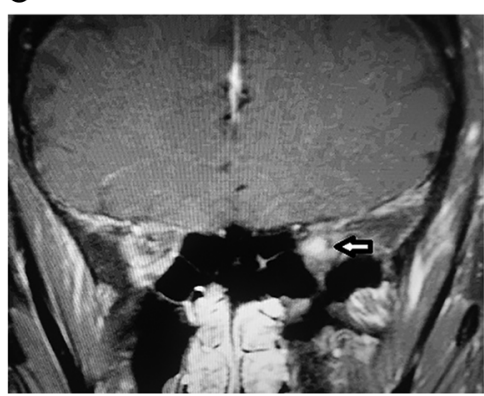

Figure 1 (A) MRI T1-weighted (T1-W) image without contrast, axial view, shows a $0.6 \mathrm{~cm} \times 0.6 \mathrm{~cm} \times 1.2 \mathrm{~cm}$ hypointense lesion (arrow) extending around the opening of the left superior orbital fissure and optic canal, lying in close proximity to the optic nerve, which is suspicious of neurogenic tumour. The optic nerve is medially displaced with buckling noted. (B) MRI T1-W fat-saturation image with contrast, axial view, shows the lesion has significant contrast enhancement (arrow). (C) MRI T1-W fat-saturation image with contrast, coronal view, shows the lesion has significant contrast enhancement (arrow). 
Table 1 Nerve conduction study

\begin{tabular}{|c|c|c|c|c|c|c|c|c|}
\hline Nerve and site & Latency & Amplitude & \multicolumn{2}{|l|}{ Segment } & CMAP duration & \multicolumn{2}{|c|}{ CMAP area } & Conduction velocity \\
\hline \multicolumn{9}{|c|}{ Motor nerve conduction } \\
\hline \multicolumn{9}{|c|}{ Median $\mathrm{R}$} \\
\hline Wrist & $5.7 \mathrm{~ms}(\mathrm{~N}<4)$ & $5.6 \mathrm{mV}(\mathrm{N}>5.6)$ & \multicolumn{2}{|c|}{ Abductor pollicis brevis-wrist } & $10.5 \mathrm{~ms}$ & \multicolumn{2}{|c|}{$20.3 \mathrm{mVms}$} & $\mathrm{m} / \mathrm{s}(\mathrm{N}>47)$ \\
\hline Elbow & $29.2 \mathrm{~ms}$ & $2.0 \mathrm{mV}$ & \multicolumn{2}{|c|}{ Wrist-elbow } & $8.9 \mathrm{~ms}(-15 \%)$ & \multicolumn{2}{|c|}{$3.7 \mathrm{mVms}(-82 \%)^{*}$} & $10 \mathrm{~m} / \mathrm{s}$ \\
\hline \multicolumn{9}{|l|}{ Ulnar R } \\
\hline Wrist & $5.1 \mathrm{~ms}(\mathrm{~N}<3.9)$ & $5.7 \mathrm{mV}(\mathrm{N}>4.5)$ & \multicolumn{2}{|c|}{ Abductor digiti minimi (manus)-wrist } & $7.0 \mathrm{~ms}$ & \multicolumn{2}{|c|}{$15.2 \mathrm{mVms}$} & $\mathrm{m} / \mathrm{s}(\mathrm{N}>47)$ \\
\hline Below the elbow & $15.7 \mathrm{~ms}$ & $1.8 \mathrm{mV}$ & Wrist-belo & he elbow & $7.0 \mathrm{~ms}(+0 \%)$ & $4.0 \mathrm{~m}$ & s $(-74 \%)^{*}$ & $21 \mathrm{~m} / \mathrm{s}$ \\
\hline Above the elbow & $17.9 \mathrm{~ms}$ & $1.4 \mathrm{mV}$ & Below the & ow-above the elbow & $9.0 \mathrm{~ms}(+28 \%)$ & $2.3 \mathrm{~m}$ & s $(-42.5 \%)^{*}$ & $36 \mathrm{~m} / \mathrm{s}$ \\
\hline Tibial R & & & & & & & & \\
\hline Ankle & $9.1 \mathrm{~ms}(\mathrm{~N}<6.3)$ & $3.7 \mathrm{mV}(\mathrm{N}>5.9)$ & Abductor I & ucis-ankle & $8.2 \mathrm{~ms}$ & $10.5 n$ & & $\mathrm{~m} / \mathrm{s}(\mathrm{N}>44)$ \\
\hline Popliteal fossa & $30.1 \mathrm{~ms}$ & $1.6 \mathrm{mV}$ & Ankle-pop & al fossa & $10.8 \mathrm{~ms}(+32 \%)$ & $7.6 \mathrm{~m}$ & $s(-31 \%)$ & $19 \mathrm{~m} / \mathrm{s}$ \\
\hline Peroneal R & & & & & & & & \\
\hline Ankle & $5.0 \mathrm{~ms}(\mathrm{~N}<5.9)$ & $0.9 \mathrm{mV}(\mathrm{N}>2.3)$ & Extensor d & orum brevis-ankle & $8.9 \mathrm{~ms}$ & $2.4 \mathrm{~m}$ & & $\mathrm{~m} / \mathrm{s}(\mathrm{N}>43)$ \\
\hline Fibula (head) & $13.9 \mathrm{~ms}$ & $1.1 \mathrm{mV}$ & Ankle-fibu & head) & $12.6 \mathrm{~ms}(+42 \%)$ & $4.0 \mathrm{~m}$ & s (+40\%) & $34 \mathrm{~m} / \mathrm{s}$ \\
\hline Popliteal fossa & $19.7 \mathrm{~ms}$ & $0.8 \mathrm{mV}$ & Fibula (he & popliteal fossa & $15.1 \mathrm{~ms}(+20 \%)$ & $3.2 \mathrm{~m}$ & s $(-20 \%)$ & $10 \mathrm{~m} / \mathrm{s}$ \\
\hline Tibial L & & & & & & & & \\
\hline Ankle & $5.3 \mathrm{~ms}(\mathrm{~N}<6.3)$ & $10.5 \mathrm{mV}(\mathrm{N}>5.9)$ & Abductor I & ucis-ankle & $8.2 \mathrm{~ms}$ & $23.9 n$ & & $\mathrm{~m} / \mathrm{s}(\mathrm{N}>44)$ \\
\hline Popliteal fossa & $20.2 \mathrm{~ms}$ & $2.7 \mathrm{mV}$ & Ankle-pop & al fossa & $9.8 \mathrm{~ms}(+20 \%)$ & $7.1 \mathrm{~m}$ & s $(-70 \%)^{*}$ & $27 \mathrm{~m} / \mathrm{s}$ \\
\hline Peroneal L & & & & & & & & \\
\hline Ankle & $5.6 \mathrm{~ms}(\mathrm{~N}<5.9)$ & $1.6 \mathrm{mV}(\mathrm{N}>2.3)$ & Extensor d & orum brevis-ankle & $7.2 \mathrm{~ms}$ & $4.3 \mathrm{~m}$ & & $\mathrm{~m} / \mathrm{s}(\mathrm{N}>43)$ \\
\hline Fibula (head) & $15.0 \mathrm{~ms}$ & $1.7 \mathrm{mV}$ & Ankle-fibu & head) & $7.5 \mathrm{~ms}(+4 \%)$ & $4.3 \mathrm{~m}$ & s $(-0 \%)$ & $32 \mathrm{~m} / \mathrm{s}$ \\
\hline Popliteal fossa & $15.9 \mathrm{~ms}$ & $1.7 \mathrm{mV}$ & Fibula (he & popliteal fossa & $7.7 \mathrm{~ms}(+3 \%)$ & $4.1 \mathrm{~m}$ & s $(-5 \%)$ & $44 \mathrm{~m} / \mathrm{s}$ \\
\hline Median L & & & & & & & & \\
\hline Wrist & $8.1 \mathrm{~ms}(\mathrm{~N}<4)$ & $3.2 \mathrm{mV}(\mathrm{N}>5.6)$ & Abductor & icis brevis-wrist & $5.9 \mathrm{~ms}$ & $5.7 \mathrm{~m}$ & & $\mathrm{~m} / \mathrm{s}(\mathrm{N}>47)$ \\
\hline Elbow & $20.8 \mathrm{~ms}$ & $2.1 \mathrm{mV}$ & Wrist-elbo & & $6.3 \mathrm{~ms}(+7 \%)^{*}$ & $4.2 \mathrm{~m}$ & s $(-26 \%)$ & $16 \mathrm{~m} / \mathrm{s}$ \\
\hline Ulnar L & & & & & & & & \\
\hline Wrist & $4.4 \mathrm{~ms}(\mathrm{~N}<3.9)$ & $5.0 \mathrm{mV}(\mathrm{N}>4.5)$ & Abductor & ti minimi (manus)-wrist & $6.2 \mathrm{~ms}$ & $10.7 n$ & & $\mathrm{~m} / \mathrm{s}(\mathrm{N}>47)$ \\
\hline Below the elbow & $12.7 \mathrm{~ms}$ & $1.2 \mathrm{mV}$ & Wrist-belo & he elbow & $12.9 \mathrm{~ms}(+108 \%)$ & $6.6 \mathrm{~m}$ & $S(-38 \%)^{*}$ & $22 \mathrm{~m} / \mathrm{s}$ \\
\hline Above the elbow & $18.3 \mathrm{~ms}$ & $2.6 \mathrm{mV}$ & Below the & ow-above the elbow & $10.1 \mathrm{~ms}(-22 \%)$ & $7.3 \mathrm{~m}$ & $\mathrm{~s}(+11 \%)$ & $38 \mathrm{~m} / \mathrm{s}$ \\
\hline Nerve and site & Onset latency & Peak latency & Amplitude & Segment & Latency dif & erence & Distance & Conduction velocity \\
\hline Sensory nerve conds & ction & & & & & & & \\
\hline Ulnar L & & & & & & & & \\
\hline Wrist & NR ms & NR ms & NR $\mu \mathrm{V}$ & Digit V (little finger)-Wrist & ms & & $140 \mathrm{~mm}$ & $\mathrm{~m} / \mathrm{s}$ \\
\hline Median L & & & & & & & & \\
\hline Wrist & NR ms & NR ms & $\mathrm{NR} \mu \mathrm{V}$ & Digit II (index finger)-Wrist & ms & & $140 \mathrm{~mm}$ & $\mathrm{~m} / \mathrm{s}$ \\
\hline Sural L & & & & & & & & \\
\hline Lower leg & $\mathrm{NR} \mathrm{ms}$ & NR ms & $\mathrm{NR} \mu \mathrm{V}$ & Ankle-Lower leg & ms & & $140 \mathrm{~mm}$ & $\mathrm{~m} / \mathrm{s}$ \\
\hline Sural R & & & & & & & & \\
\hline Lower leg & NR ms & NR ms & $\mathrm{NR} \mu \mathrm{V}$ & Ankle-Lower leg & ms & & $140 \mathrm{~mm}$ & $\mathrm{~m} / \mathrm{s}$ \\
\hline Ulnar R & & & & & & & & \\
\hline Wrist & NR ms & NR ms & $\mathrm{NR} \mu \mathrm{V}$ & Digit V (little finger)-Wrist & ms & & $140 \mathrm{~mm}$ & $\mathrm{~m} / \mathrm{s}$ \\
\hline Median R & & & & & & & & \\
\hline Wrist & NR ms & NR ms & $\mathrm{NR} \mu \mathrm{V}$ & Digit II (index finger)-wrist & ms & & $140 \mathrm{~mm}$ & $\mathrm{~m} / \mathrm{s}$ \\
\hline Nerve & & M late & ncy & & F latency & & & F wave $\%$ \\
\hline$F$ wave studies & & & & & & & & \\
\hline Median L & & 6.5 & & & & & & 0.0 \\
\hline Ulnar L & & 4.6 & & & $24.4(\mathrm{~N}<28)$ & & & 90.0 \\
\hline Tibial L & & 5.4 & & & $99.9(\mathrm{~N}<55)$ & & & 10.0 \\
\hline Peroneal L & & 5.1 & & & & & & 0.0 \\
\hline Tibial R & & 8.9 & & & $56.5(\mathrm{~N}<55)$ & & & 10.0 \\
\hline Peroneal R & & 5.7 & & & & & & 0.0 \\
\hline Median R & & 7.3 & & & & & & 0.0 \\
\hline Ulnar R & & 5.0 & & & & & & 0.0 \\
\hline
\end{tabular}


Figure 2 (A) MRI T2-weighted (T2-W) image short T1 inversion recovery (STIR) sequence, coronal view, shows diffuse thickening of cervical nerve roots (arrows). (B) MRI T2-W image STIR sequence, coronal view, shows diffuse thickening of the brachial plexuses (arrows).
A

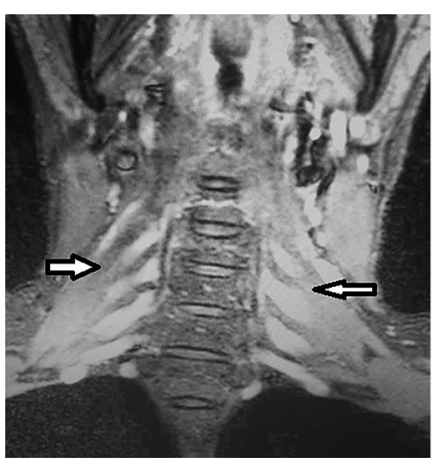

B

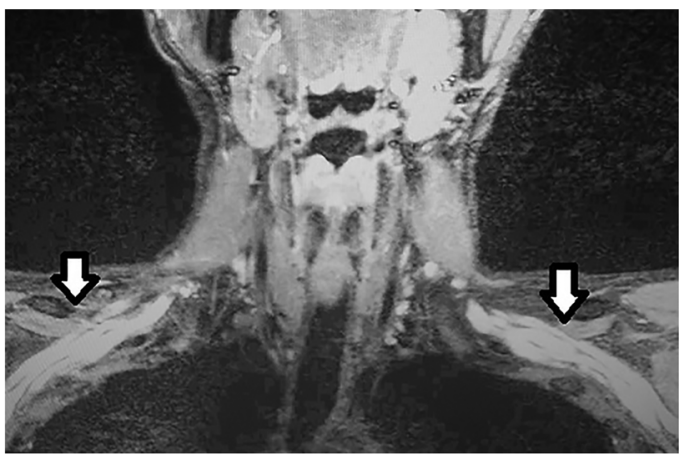

Competing interests None.

Patient consent Obtained.

Provenance and peer review Not commissioned; externally peer reviewed.

\section{REFERENCES}

1 Olney RK. Guidelines in electrodiagnostic medicine. Consensus criteria for the diagnosis of partial conduction block. Muscle Nerve Supp/ 1999;8:S225-9.

2 Gunalp I, Gunduz K, Duruk K, et al. Neurogenic tumors of the orbit. Jpn J Ophthalmol 1994;38:185-90.

Copyright 2014 BMJ Publishing Group. All rights reserved. For permission to reuse any of this content visit http://group.bmi.com/group/rights-licensing/permissions.

BMJ Case Report Fellows may re-use this article for personal use and teaching without any further permission.

Become a Fellow of BMJ Case Reports today and you can:

- Submit as many cases as you like

- Enjoy fast sympathetic peer review and rapid publication of accepted articles

- Access all the published articles

- Re-use any of the published material for personal use and teaching without further permission

For information on Institutional Fellowships contact consortiasales@bmjgroup.com

Visit casereports.bmj.com for more articles like this and to become a Fellow 